Revue internationale P.M.E.

Économie et gestion de la petite et moyenne entreprise

\title{
Modifications récentes de la fonction logistique chez les PME exportatrices et non exportatrices québécoises
}

\section{Yvon Bigras}

Volume 7, numéro 2, 1994

URI : https://id.erudit.org/iderudit/1008391ar

DOI : https://doi.org/10.7202/1008391ar

Aller au sommaire du numéro

Éditeur(s)

Presses de l’Université du Québec

ISSN

0776-5436 (imprimé)

1918-9699 (numérique)

Découvrir la revue

Citer cette note

Bigras, Y. (1994). Modifications récentes de la fonction logistique chez les PME exportatrices et non exportatrices québécoises. Revue internationale P.M.E., 7(2), 105-116. https://doi.org/10.7202/1008391ar

\section{Résumé de l'article}

La globalisation de l'économie, la déréglementation dans le secteur des transports et le développement de nouveaux outils informatiques sont autant de phénomènes qui se sont conjugués pour amorcer une modification profonde des besoins logistiques de l'entreprise. Dans cette note de recherche, nous examinerons, à partir d'un échantillon de 319 établissements, comment les PME québécoises se sont adaptées à ce nouvel environnement.

L'étude fera d'abord ressortir que la déréglementation du transport routier au Canada, en 1988, a effectivement amélioré la qualité et la diversité des prestations de services offerts aux PME. Ces changements se sont également accompagnés de pressions à la baisse sur les prix du transport, situation dont ont pu profiter les PME de toutes les régions du Québec. Nous démontrons par la suite que cela a d'abord eu des répercussions sur la nature de leurs relations avec les prestataires de services de transport, mais également sur les autres éléments de leur fonction logistique. Finalement, il semble que les PME exportatrices réagissent plus fortement à ces changements dans leur environnement et qu'elles ont tendance à modifier les différents éléments de leur fonction logistique. Cela s'expliquerait par les pressions concurrentielles plus fortes qui s'exercent sur elles.
Ce document est protégé par la loi sur le droit d'auteur. L'utilisation des services d'Érudit (y compris la reproduction) est assujettie à sa politique d'utilisation que vous pouvez consulter en ligne.

https://apropos.erudit.org/fr/usagers/politique-dutilisation/ 


\title{
Modifications récentes de la fonction logistique chez les PME exportatrices et non exportatrices québécoises
}

Yvon BIGRAS*

Université du Québec à Trois-Rivières

\begin{abstract}
RÉSUMÉ
La globalisation de l'économie, la déréglementation dans le secteur des transports et le développement de nouveaux outils informatiques sont autant de phénomènes qui se sont conjugués pour amorcer une modification profonde des besoins logistiques de l'entreprise. Dans cette note de recherche, nous examinerons, à partir d'un échantillon de 319 établissements, comment les PME québécoises se sont adaptées à ce nouvel environnement.

L'étude fera d'abord ressortir que la déréglementation du transport routier au Canada, en 1988, a effectivement amélioré la qualité et la diversité des prestations de services offerts aux PME. Ces changements se sont également accompagnés de pressions à la baisse sur les prix du transport, situation dont ont pu profiter les PME de toutes les régions du Québec. Nous démontrons par la suite que cela a d'abord eu des répercussions sur la nature de leurs relations avec les prestataires de services de transport, mais également sur les autres éléments de leur fonction logistique. Finalement, il semble que les PME exportatrices réagissent plus fortement à ces changements dans leur environnement et qu'elles ont tendance à modifier les différents éléments de leur fonction logistique. Cela s'expliquerait par les pressions concurrentielles plus fortes qui s'exercent sur elles.
\end{abstract}

* Yvon Bigras est professeur d'économique au Département d'administration et d'économique de l'Université du Québec à Trois-Rivières et membre du Groupe de recherche en économie et gestion des PME (GREPME). Il est détenteur d'un Ph. D. en sciences économiques obtenu au Centre de recherche sur les transports de l'Université de Montréal. Adresse: GREPME, Université du Québec à Trois-Rivières, C.P. 500, Trois-Rivières (Québec) G9A 5H7. 


\section{ABSTRACT}

The globalization of the economy, the economic deregulation in the sector of transportation and the development of new information management supports, are phenomenons that are all pushing towards a revolution in SMEs' logistic needs. In this research note we will try to find from a sample of 319 establishments, how Quebec's SMEs are adapting to this new environment.

The paper will first emphasize on the positive benefits of trucking deregulation in Canada, starting in 1988, both on the quality and the diversity of the services provided by the trucking industry. These changes come while competitive pressures were keeping the price of transportation low. SMEs, even in remote regions of Quebec, benefitted from this situation. We then show how this new environment has changed the kind of relations between shippers and carriers, but also how it changed other aspects of the whole logistic function of the firm. Finally, it seems that exporting SMEs are adjusting more quickly and more deeply their logistic function than non exporting one. Stronger competitive pressures on markets where exporting SMEs are involved can explain this situation.

\section{RESUMEN}

El crecimiento de la interdependencia de las economías, la desregulacion del sector de transporte y el desarollo de nuevos equipos informáticos son algunos de los fenómenos, que aunados, llevan al cabo una modificación profunda de las necesidades logísticas de las empresas. En este apunte de investigación examinaremos, a partir de una muestra formada por 319 establecimientos, como la PME quebequense se ha adaptado a este nuevo medio ambiente.

Este estudio mostrará antes que todo como la desregulación del autotransporte canadiense, realizada en 1988, ha realmente mejorado la calidad y la diversidad de las prestaciones de servicios de transporte ofrecidas a las PME. Estos cambios han sido acompañados a su vez de presiones a la baja sobre los precios de transporte, situacion de la cual las PME de todas las regionas de Quebec han sabido muy bien aprovechar. Por consiguiente, demostramos que toda esta situación ha tenido repercuciones sobre la naturaleza de la relaciones establecidas entre las empresas y los prestatarios de servicios de transporte, de la misma forma en que ha repercutido también sobre todos los demás elementos de la función logística de las empresas. Finalmente, parece ser que las PME exportadoras reaccionan de manera más intensa a estos cambios dentro de su medio ambiente y que son más propensas a modificar los diferentes elementos de su función logística. Esta situación puede explicarse por las presiones, cada vez más fuertes, que la competencia ejerce sobre estas PME.

\section{Introduction}

L'interdépendance accrue des économies, la déréglementation dans les transports et le développement d'outils informatiques sont autant de phénomènes qui se sont conjugués au cours des dix dernières années pour amorcer une véritable révolution des besoins logistiques de l'entreprise. Pour les PME, les nouvelles 
exigences logistiques qui résultent de l'ouverture des marchés posent plusieurs défis. La logistique de l'entreprise devenant un outil stratégique de plus en plus important, les PME doivent modifier leurs politiques et pratiques logistiques afin de profiter au maximum des occasions offertes par la déréglementation des transports et l'informatisation accrue.

Dans cette note, nous explorerons certaines hypothèses permettant de comprendre la nature des changements en cours dans les PME québécoises. Nous présenterons d'abord le cadre théorique qui nous a guidé dans notre analyse, la méthodologie utilisée, et en particulier, l'échantillon de PME étudié, pour ensuite examiner les trois questions suivantes:

1. La déréglementation a-t-elle donné accès aux PME à des services bien adaptés à leurs besoins, de qualité et à des coûts compétitifs ?

2. Les PME ont-elles adapté leur fonction logistique en profitant des occasions offertes par la déréglementation?

3. La concurrence internationale est-elle un déclencheur de ces ajustements logistiques?

\section{Cadre théorique}

\subsection{Impact de la déréglementation}

La déréglementation du transport a facilité, voire permis, certains des ajustements logistiques apportés par les entreprises. Nous voulons donc d'abord vérifier quel a été l'impact pour les PME de cette déréglementation en insistant principalement sur le cas du transport routier qui touche le plus directement les PME. La question de l'impact économique global de la déréglementation a été abondamment traitée par la littérature sur la déréglementation du transport routier aux États-Unis (Bailey, 1981; Friedlaender et Spady, 1981; Harmatuck, 1981; Wang Chiang et Friedlaender, 1985). On retrouve la même discussion au sujet de l'industrie canadienne et québécoise du camionnage (Bigras, 1993; Boucher, 1988; Gagné et Dionne, 1988).

Cette littérature fait ressortir deux questions cruciales pour l'évolution de cette industrie et de sa performance en termes de tarifs et de qualité des services. D'abord, les rendements d'échelle caractérisant cette industrie sont-ils croissants, décroissants ou constants? S'ils sont croissants et que l'on n'a pas épuisé ces économies d'échelle, il pourrait en résulter une concentration excessive après quelques épisodes de concurrence destructrice. Les études américaines font ressortir que, s'il y a des économies d'échelle, elles ne sont pas très importantes et ne sont pas reliées à la taille des installations. Elles découlent en fait d'économies de champ (economies of scope) associées à la production simultanée de plusieurs produits. 
Pour l'industrie québécoise du camionnage, Boucher (1988) et Dionne et Gagné (1988), malgré des méthodologies différentes, arrivent à des prévisions assez analogues dans la mesure où ils ne prévoient ni concentration excessive et ni concurrence destructrice pour l'ensemble de l'industrie. Selon Boucher, il y aura certains ajustements en raison d'économies de champ non complètement épuisées. Pour Gagné et Dionne, les plus gros transporteurs ont des rendements d'échelle constants et les ajustements devraient se limiter aux plus petits transporteurs. Globalement, on ne devrait donc pas assister à une concentration excessive dans ce secteur. Au contraire, il faut prévoir une amélioration de la productivité qui devrait se traduire par des prix plus bas et une meilleure qualité des services. Globalement, ces prévisions optimistes semblent s'être réalisées selon Bigras (1993).

La deuxième question concerne la discrimination possible dont pourrait être victime les petits expéditeurs et les expéditeurs dans les régions périphériques. La question est alors de savoir dans quelle mesure ces expéditeurs profitaient d'un interfinancement positif avant la déréglementation, situation qui allait disparaître avec la déréglementation sous la pression d'une concurrence accrue sur les marchés principaux. Là-dessus, les avis étaient partagés. Boucher (1985) prévoyait qu'au Québec les petits expéditeurs ne seraient pas pénalisés puisqu'ils ne bénéficiaient pas vraiment de subventions déguisées. Aux ÉtatsUnis, c'est ce que l'on a observé si l'on se fie à l'analyse des différentes études faites par Harper et Jonhson (1987), bien que les avis demeurent encore partagés (Athearn, 1991).

\subsection{Impact sur la fonction logistique}

Si l'on considère l'ensemble de la fonction logistique de façon intégrée, des modifications dans la qualité, la diversité et le prix des prestations de services de transport routier doivent induire des ajustements dans les autres éléments de la fonction logistique. On peut déduire cette relation à la fois de l'approche du coût total de la distribution physique (Christopher, 1985) ou de l'approche de la chaîne de valeur (Porter, 1986). Il faut cependant souligner que les ajustements à la fonction logistique, en même temps qu'ils profitent de la déréglementation, doivent répondre à de nouvelles exigences logistiques. Colin et Paché (1988) caractérisent celles-ci par les deux exigences de fluidité dans l'écoulement des marchandises et la continuité de la chaîne logistique. Cela implique que non seulement les délais de livraison doivent être raccourcis mais, en plus, ils doivent être d'une fiabilité absolue et au coût le plus bas possible afin d'assurer la compétitivité des produits sur des marchés de plus en plus éloignés. Il sera évidemment impossible de départager dans quelle mesure les changements logistiques répondent aux nouvelles exigences logistiques ou aux modifications de l'offre de services de transport, les deux mouvements étant en fait interdépendants. 
On veut finalement vérifier l'hypothèse que les PME impliquées dans le commerce international réagissent plus rapidement et sont plus nombreuses à modifier leur fonction logistique. La concurrence étant plus vive sur des marchés ouverts, les pressions pour adopter le plus rapidement possible les sources davantage concurrentielles sont plus fortes. Cette relation s'appuie sur le modèle de Porter (1986) des forces concurrentielles. En effet, les pressions subies par la PME étant plus fortes, elle doit développer ses stratégies face à la concurrence plus rapidement. Ce modèle fait également ressortir l'importance de la relation entre l'entreprise et ses fournisseurs. Or, cette relation a certainement été modifiée par la déréglementation des transports et l'apparition de prestataires de services d'envergure mondiale.

\section{Méthodologie}

Les données que nous utiliserons proviennent d'une enquête réalisée pour le compte du ministère des Transports du Québec en 1991 (Bigras, 1991). Cette enquête concernait les activités de distribution et de transport des entreprises dans le contexte de la déréglementation du transport au Canada le $1^{\text {er }}$ janvier 1988. Le questionnaire porte sur les services de transport routier, mais dans le cadre d'ensemble de la fonction logistique de l'entreprise.

Au total, nous avons recueilli des informations par un sondage postal auprès de 665 établissements dans les secteurs manufacturier et du commerce de gros, en plus de réaliser 23 entrevues. On a procédé à un échantillonnage aléatoire stratifié dans la population de 11694 établissements manufacturiers, lequel était divisé en strates selon la taille des établissements (PME et grands établissements) et leur situation géographique (Montréal, régions centrales et régions périphériques). Comme la proportion de réponses varie d'une strate à l'autre, on associe alors un poids à chaque répondant pour corriger le biais causé par les taux d'échantillonnage différents entre les strates.

Pour l'analyse présentée ici, nous nous sommes concentré sur les 319 établissements manufacturiers de moins de 200 employés. On retrouve parmi ceux-ci 102 établissements qui exportaient une partie de leur production à l'extérieur du Canada, alors que les 217 autres écoulaient toute leur production à l'intérieur du Canada. La proportion de 32,0\% d'établissements qui exportent est très élevée. Évidemment, il est difficile de comparer ces données avec d'autres (Joyal et al., 1993) parce que la population visée n'est pas exactement la même. En effet, la population cible de notre étude regroupait les établissements et non les entreprises. Nous considérions que ce niveau est plus approprié pour évaluer la qualité des prestations de services de transport. 


\section{Résultats}

\subsection{Déréglementation et concurrence dans les transports}

Si l'on analyse ce qui s'est passé dans le transport routier au Canada depuis la déréglementation du $1^{\text {er }}$ janvier 1988, on constate que l'offre de transport a été caractérisée par deux mouvements opposés. D'une part, il y a eu un mouvement de concentration dans l'industrie qui s'est traduit par des faillites, des acquisitions ou l'abandon des affaires. D'autre part, certains transporteurs ont étendu leur aire de marché au-delà de ce que permettaient leurs anciens permis.

Pour l'instant, il semble bien que le deuxième mouvement ait pris le dessus et permis une hausse de la concurrence sur les différents marchés. En effet, la grande majorité des établissements interrogés considèrent que le nombre de transporteurs offrant leur service a augmenté. Sur les six segments de marché examinés (charges complètes et groupage routier, sur les plans intraprovincial, interprovincial et international), il n'y a jamais plus du quart des répondants qui considèrent que le nombre de transporteurs a diminué. Si l'on se concentre sur les marchés internationaux, au regard du groupage routier, seulement $12,8 \%$ des établissements considèrent que le nombre de concurrents a diminué et, sur le marché des charges complètes, ce pourcentage n'est que de $21,6 \%$. Les données nous permettent, par ailleurs, d'ajouter que cette augmentation de la concurrence s'est vérifiée autant à Montréal que dans les régions centrales et périphériques (Bigras et Pettigrew, 1992).

Cette augmentation de la concurrence a amené de fortes pressions à la baisse sur les tarifs. Si l'on calcule l'augmentation totale des coûts unitaires de transport entre le $1^{\mathrm{er}}$ janvier 1988 et le $1^{\text {er }}$ juin 1991, soit une période de 41 mois, on constate que sur tous les segments de marché, le nombre d'établissements ayant subi une hausse de plus de $10 \%$ est très faible. La grande majorité des établissements ont connu une hausse de moins de $10 \%$, voire une baisse des prix de transport. Ainsi, pour ce qui est des flux extraprovinciaux de charges complètes par camion, seulement $8,7 \%$ des établissements ont connu une hausse supérieure à $10 \%$ pendant toute cette période.

Dans ces conditions, est-ce que les petits et moyens établissements ont pu obtenir des services mieux adaptés aux nouvelles exigences logistiques? Sans être une indication directe, les niveaux de satisfaction très élevés du tableau 1 vont dans ce sens. En effet, $20,8 \%$ des établissements se sont dits « très satisfaits » et $75,5 \%$ se sont dits « assez satisfaits » des services offerts par les transporteurs routiers avec lesquels ils faisaient affaires. Cela donne un pourcentage très élevé de $96,3 \%$, lequel est corroboré par l'Office national des transports (1992) pour l'ensemble du Canada. De plus, cette satisfaction s'est accrue au cours des dernières années. En effet, une majorité des répondants pensent que les services se sont améliorés. 
TABleau 1

Satisfaction quant aux services offerts par les transporteurs (\% des établissements)

\begin{tabular}{lr}
\hline Très satisfait & 20,8 \\
Assez satisfait & 75,5 \\
Peu satisfait & 2,7 \\
Pas du tout satisfait & 1,0 \\
\hline
\end{tabular}

Ce sont tous les aspects des services qui se sont améliorés, que ce soit les temps de transit, la fréquence des cueillettes, la disponibilité des équipements, le suivi de l'expédition, etc. Les transporteurs doivent donc maintenant offrir des services complets en Amérique du Nord et ils ont rationalisé leurs opérations en établissant des réseaux en étoile, des itinéraires triangulaires, ou en s'alliant avec d'autres transporteurs. L'élimination des permis a ainsi ouvert la porte à une rationalisation des opérations qui se mesure en particulier par la diminution des retours à vide (Office national des transports, 1992).

\subsection{Les nouvelles relations avec les prestataires de services}

Le nouvel environnement créé par la déréglementation a amené les expéditeurs à modifier certaines politiques et pratiques. La première concerne leurs relations avec les transporteurs. C'est ce que l'on retrouve au tableau 2, lequel fait en plus la distinction entre les établissements exportateurs et non-exportateurs. On y note que $87,7 \%$ des établissements exportateurs et $69,2 \%$ des établissements non exportateurs déclarent avoir changé certains des transporteurs avec lesquels ils faisaient affaire de façon régulière. La raison principale pour justifier cette infidélité: les tarifs dans la majorité des cas; mais, pour les exportateurs, la qualité des services arrive tout près derrière avec $44,0 \%$, alors que pour les non-exportateurs ce pourcentage n'est que de $30,5 \%$. Cela implique également que les établissements doivent évaluer fréquemment la performance de leurs transporteurs, surtout si on est un exportateur. Cette tendance semble se confirmer puisque $52,0 \%$ des exportateurs déclarent évaluer « souvent » la performance de leurs transporteurs contre $37,7 \%$ des non-exportateurs.

Cette infidélité des expéditeurs est tout à fait nouvelle car, dans un environnement réglementé, ceux-ci devaient accepter les services que les transporteurs ayant la permission d'opérer sur leur territoire voulaient bien leur consentir. Les transporteurs s'assuraient alors la fidélité de leur clientèle en établissant avec eux des liens personnels plutôt paternalistes. On confiait pendant des années ses expéditions au même transporteur, sans vraiment évaluer sa performance, qui de toute façon variait bien peu d'un transporteur à l'autre. Ces vieilles relations de confiance se sont écroulées avec l'ouverture de ce secteur à la concurrence. 


\section{TABleau 2 \\ Établissements ayant changé certains de leurs transporteurs réguliers et raisons invoquées}

\begin{tabular}{lcc}
\hline & Exportateurs & Non exportateurs \\
Pourcentage des établissements ayant changé & 87,7 & 69,2 \\
Principale raison invoquée si changement (en \%) & & \\
Tarifs & 53,3 & 55,3 \\
Services & 44,0 & 30,5 \\
Nouveaux besoins & 0,1 & 10,4 \\
Autres raisons & 2,6 & 3,8 \\
\hline
\end{tabular}

Il s'en suit également que le nombre de transporteurs avec lesquels on fera affaire diminuera. L'Office national des transports (1992) note en effet que près de quatre expéditeurs sur dix affirment avoir réduit le nombre de transporteurs avec qui ils font affaire. Cela correspond à la stratégie d'un nombre grandissant de transporteurs qui tentent d'obtenir une part de plus en plus grande des marchandises d'un client donné en leur offrant des services à valeur ajoutée d'entreposage et de planification logistique.

\subsection{La fonction logistique}

La diversité des services offerts dans un environnement déréglementé, la possibilité de négocier avec le fournisseur des services de transport et les exigences accrues des marchés ouverts semblent avoir provoqué une véritable révolution des pratiques logistiques dans l'entreprise.

Le tableau 3 indique que de janvier 1988 à juin 1991, 65,2\% des établissements exportateurs déclarent avoir modifié leur façon de gérer les stocks. Pour les établissements non exportateurs, le pourcentage est également élevé, mais moindre, soit $51,5 \%$. Ce résultat va dans le sens de l'hypothèse que l'ouverture des marchés incite les entreprises à une plus grande rationalisation de la fonction logistique. Il faut cependant être prudent, car il n'est pas exclu que cette plus grande rationalisation soit en fait le lot d'entreprises qui, parce qu'elles sont plus performantes, sont présentes sur les marchés extérieurs. Le résultat tend au moins à confirmer un lien entre l'ouverture des marchés et la rationalisation de la fonction logistique.

Pour les deux groupes d'établissements, la principale raison invoquée pour expliquer ces changements est la volonté de diminuer le niveau des stocks ( $65,1 \%$ chez les exportateurs et $68,7 \%$ chez les non-exportateurs). Pour le reste, il y a quelques différences à noter, en particulier l'importance plus grande 
accordée par les établissements exportateurs à la nécessité de s'adapter à un système de production «juste-à-temps ». Dans le cas des exportateurs, le « juste-à-temps » est cité par 54,3\% de ceux qui ont apporté des changements, alors que ce pourcentage n'est que de 33,2\% pour les non-exportateurs. La pratique du «flux tendu » n'est plus marginale, mais de plus, la présence sur les marchés extérieurs semble être reliée à un plus grand recours à cette pratique. Cela est évidemment possible dans la mesure où l'on assiste à une intégration mondiale des systèmes de transport, dont l'une des caractéristiques est le service « autour du monde ».

TABLEAU 3

Modifications dans la façon de gérer les stocks

\begin{tabular}{lcc}
\hline & Exportateurs & Non exportateurs \\
Pourcentage des établissements ayant modifié & 65,2 & 51,5 \\
leur façon de gérer leurs stocks & & \\
Raisons invoquées si changement (en \%) & 65,1 & 68,7 \\
Diminuer les stocks & 6,1 & 4,4 \\
Accroître les stocks & 54,3 & 33,2 \\
S'adapter au « juste-à-temps » & 49,6 & 31,1 \\
Augmenter la rotation des stocks & 15,1 & 12,3 \\
Augmentation de la demande & 11,7 & 14,2 \\
Baisse de la demande & 12,5 & 24,4 \\
Changements de gamme de produits & & \\
\hline
\end{tabular}

Le tableau 4, qui porte sur la connexion à un réseau informatisé de communication, fait également ressortir une différence importante entre les établissements exportateurs et non exportateurs. Les premiers sont reliés à un tel réseau dans $24,9 \%$ des cas et utilisent plus intensivement cet outil, soit pour $32,7 \%$ des commandes qu'ils reçoivent. Quant aux non-exportateurs, ils ne sont reliés que dans $15,6 \%$ des cas et utilisent cet outil pour $27,0 \%$ de leurs commandes. Il est plus difficile d'interpréter ce résultat qui peut aussi bien découler des exigences accrues sur les marchés d'exportation que d'un développement plus poussé de l'EDI pour ce type d'expéditions.

TABleau 4

Établissements reliés à un réseau informatisé de communication (EDI)

\begin{tabular}{lcc}
\hline & Exportateurs & Non exportateurs \\
Pourcentage des établissements & 65,2 & 51,5 \\
$\begin{array}{l}\text { Commandes reçues par ce moyen } \\
\text { (en \% pour ceux qui sont reliés) }\end{array}$ & 32,7 & 27,0 \\
\hline
\end{tabular}


On retrouve finalement dans l'enquête une donnée qui tend à confirmer que les marchés extérieurs sont devenus plus exigeants au cours des dernières années et que les entreprises ont dû y faire des efforts plus grands que sur les marchés intérieurs. Au tableau 5, on présente les résultats d'une question qui portait sur les modifications du niveau global de services à la clientèle. Or, on note que $68,6 \%$ des exportateurs ont augmenté («fortement » ou «légèrement») ce niveau de services à la clientèle, alors que seulement $47,4 \%$ des nonexportateurs ont fait la même chose.

TABLeau 5

Modifications du niveau global de services à la clientèle

\begin{tabular}{lcc}
\hline & Exportateurs & Non exportateurs \\
A fortement augmenté & 20,9 & 20,8 \\
A légèrement augmenté & 47,7 & 26,6 \\
Est demeuré stable & 24,5 & 43,1 \\
A légèrement diminué & 3,4 & 5,8 \\
A fortement diminué & 3,6 & 3,6 \\
\hline
\end{tabular}

\section{Conclusion: les enjeux pour la PME exportatrice}

Les PME québécoises semblent donc avoir amorcé leur révolution logistique en réponse à l'ouverture des marchés. Elles ont pu profiter de la déréglementation de l'industrie du transport en Amérique du Nord qui, en augmentant la concurrence dans le secteur, a stimulé le développement de services variés, mieux adaptés à leurs besoins, d'une grande fiabilité et à des coûts très raisonnables. La diversification des services est d'ailleurs la caractéristique la plus frappante du développement des transports terrestres en Amérique du Nord au cours des dernières années. Les expéditeurs ont maintenant le choix entre une gamme de produits de configuration et de prix différents.

Cette situation n'est cependant pas sans poser des problèmes à des PME qui doivent prendre des décisions logistiques de plus en plus complexes. La maîtrise sur le transport devient un enjeu majeur. Cette maîtrise n'implique pas que la PME fasse elle-même tout le transport et la distribution de son produit, mais elle doit au moins pouvoir l'organiser ou le faire organiser « dans le sens dicté par les intérêts de l'entreprise ou conformément à sa stratégie globale » (Raharolahy, 1984). En plus de reposer sur certaines compétences internes, cela suppose surtout, dans le cas de la PME, que l'on puisse compter sur des services conseils externes bien adaptés aux besoins de l'entreprise. 
À ce sujet, apparaissent certaines lacunes quant au soutien sur lequel peuvent compter les PME québécoises. Nous ne parlerons pas ici des programmes gouvernementaux qui semblent à première vue mieux développés en Europe. Au niveau des services privés de soutien au transport et à l'exportation, les PME québécoises sont défavorisées par l'absence de tradition et le manque de compétence. Dans une étude récente sur l'industrie des services de transit au Canada (Industrie, Sciences et Technologie Canada, 1991), on fait ressortir les faiblesses structurelles de cette industrie. Sur le marché mondial, les transitaires canadiens sont très petits et ils n'ont pas les ressources nécessaires pour établir un réseau complet de bureaux à l'étranger. La faiblesse du Canada en matière de formation dans ce domaine constitue également un problème majeur pour cette industrie. Ces difficultés trouvent leur explication dans la dépendance historique du Canada envers le marché américain où il est possible d'œuvrer sans nécessairement passer par un intermédiaire, surtout pour des matières peu transformées écoulées auprès de quelques clients industriels. Cette raison historique explique également la faiblesse des maisons de commerce canadiennes (Industrie, Sciences et Technologie Canada, 1992).

Les PME devraient donc miser sur des alliances interentreprises auxquelles seraient associées les entreprises fournisseurs de services logistiques et de transport. Ces alliances devraient viser à former des réseaux d'entreprises du type défini par Paché et Paraponaris (1993). Or, au Québec, on a toujours vu les transporteurs comme de simples fournisseurs de services sur demande, sans les associer à la planification de l'entreprise. Il est urgent de changer cette mentalité à la fois chez les expéditeurs et chez les transporteurs, et de développer de véritables alliances axées sur la logistique de l'exportation des PME québécoises.

\section{Bibliographie}

ATHEARN, F. (1991), «Availability of less-than-truckload service since deregulation in the ten states bordering Canada », Proceedings, $26^{\mathrm{e}}$ Congrès annuel du Canadian Transportation Research Forum, Québec, p. 69-83.

BAILEY, E.E. (1981), «Contestability and the design of regulatory and antitrust policy », American Economic Review, vol. 71, n 2, p. 178-183.

BIGRAS, Y. (1991), Étude des effets de la déréglementation économique auprès des usagers de services de camionnage, Rapport présenté au ministère des Transports du Québec.

BIGRAS, Y. (1993), «La déréglementation de l'industrie du camionnage au Québec: de la théorie à la réalité », L'Actualité économique, vol. 69, n 3, p. 123-138. 
BigRas, Y. et D. PetTigReW (1992), «Les expéditeurs québécois et la déréglementation économique de l'industrie du camionnage », Actes $d u$ congrès, $27^{\mathrm{e}}$ Congrès annuel du Canadian Transportation Research Forum, Banff (Alberta), p. 309-317.

BOUCHER, M. (1985), «La déréglementation américaine du camionnage public et les transporteurs publics québécois : perspective économique », Exposé des communications, $20^{\mathrm{e}}$ Congrès annuel de l'Association québécoise du transport et des routes, p. 269-279.

BOUCHER, M. (1988), "Considérations empiriques sur la technologie de l'industrie québécoise du camionnage public », L'Actualité économique, vol. 64, $\mathrm{n}^{\circ} 3$, p. 361-379.

CHRISTOPHER, M. (1985), The Strategy of Distribution Management, London, Grower.

COLIN, J. et G. PACHÉ (1988), La logistique de distribution, l'avenir du marketing, Paris, Chotard et Associés Éditeurs.

FriedlaENDER, A.F. et R.H. SPADY (1981), Freight Transport Regulation, Cambridge, MA., MIT Press.

GaGnÉ, R. et G. DiOnNe (1988), «Qu'en est-il des rendements d'échelle dans les industries québécoises et ontariennes de transport par camion?», L'Actualité économique, vol. $64, \mathrm{n}^{\circ} 3$, p. 380-395.

HARMATUCK, D.J. (1981), "A motor carrier joint cost function», Journal of Transportation Economics and Policy, vol. 15, $\mathrm{n}^{\circ} 2$, p. 135-153

HARPER, D.V. et J.C. JOHNSON (1987), « The potential consequences of deregulation of transportation revisited», Land Economics, vol. 63, n 2, p. 137-146

Industrie, SCIENCES ET TECHNOLOGIE CANADA (1991), Profil de l'industrie - Transitaires, Ottawa, Direction générale des communications.

Industrie, SCiEnCes et technologie CANAda (1992), Profil de l'industrie - Maisons de commerce, Ottawa, Direction générale des communications.

Joyal, A., P.A. Julien et L. Deshaies (1993), «L'exportation des PME québécoises et l'accord de libre-échange avec les États-Unis», Revue Internationale PME, vol. $6, \mathrm{n}^{\circ} 1$, p. $67-85$.

OFFICE NATIONAL DES TRANSPORTS DU CANADA (1992), Examen annuel 1991, Ottawa, Ministère des Approvisionnements et Services Canada.

PAChÉ, G. et C. PARAPONARIS (1993), L'entreprise en réseau, Paris, Presses universitaires de France.

PORTER, M.E. (1986), L'Avantage concurrentiel, Paris, InterÉditions.

WANG CHIANG, J.S. et A.F. FRIEDLAENDER (1985), «Truck technology and efficient market structure », Review of Economics and Statistics, vol. 67, $\mathrm{n}^{\circ} 2$, p. 250-258. 